\title{
An Intelligent Controller Applied To Renewable Energy System
}

\author{
Eisa Alsakkaf \\ Department of Electrical Engineering, University of Bridgeport, Bridgeport, CT, USA
}

\begin{abstract}
In this paper, an intelligent controller applied to renewable energy system has been discussed. The attractiveness of the photovoltaic generators (PV arrays), as a renewable power source is that they produce a clean energy without running costs. This fact in addition to the continuous reduction in the PV arrays initial cost and the increase in their efficiency imply the promising future of the PV generation systems. The PI-controller gains are accurately identified and hence it proved its ability in driving the PV module to its maximum power point in terms of majority of the performance measures. This leads to a significant increase in the delivered power, especially when the direct coupling operating point is far from the maximum power one.
\end{abstract}

Keywords: Renewable, An intelligent, Photovoltaic, Energy.

\section{INTRODUCTION}

Recently the advances in the semiconductor manufacturing technology and the power electronics have allowed the PV to be considered the promising renewable power source in the industry and residential applications. For good utilization of the PV arrays, it is necessary to operate at their maximum power points (MPP's). $20 \%$.

Therefore, many different techniques have been developed to accomplish this purpose. This chapter introduces the basic concepts of the PV system and discusses the mismatch-problem between the PV array and the load by listing some solutions to that problem. The photovoltaic electrical effect (the light is converted directly into electricity) was observed initially by Edmond Becquerel in 1839 [1]. In 1954 a solar cell with efficiency $6 \%$ was developed at Bell laboratories and saw its first application in 1958 with satellites. These cells were made by hand and cost $\$ 1000$ per watt. In 1987 the cost of mass production modules has dropped to $\$ 4-5$ per watt. Now PV is more economical for a number of terrestrial applications ranging from a few micro watts to tens of megawatts. PV power sources have become available in the last few years for many applications and markets around the world. They become popular for consumer products such as pocket calculators and many rural applications, notably small residential PV sources and solar water pumping systems [2]. The total PV capacity produced worldwide as of 1990 amounts to approximately $45 \mathrm{MW}$. Over $50 \mathrm{MW}$ of PV power modules were produced in 1991. The production rate has been increasing at almost $20 \%$ annually over the last few years, and this trend is likely to continue.

Many different solar cells are now available on the market, and more are under development. The range of solar cells spans different materials and different structures in the quest to extract maximum power of the device while keeping the cost to a minimum value. Devices with efficiency exceeding $35 \%$ have been demonstrated in the laboratory while, the efficiency of commercial devices is usually less than $50 \%$ of this value. The crystalline silicon cells hold the largest part of the market. To reduce the cost, these cells are now often made from polycrystalline material, rather than from the more expensive single crystals. Crystalline silicon cell technology is well established. The modules have a long lifetime ( 20 years or more) and their best production efficiency is approaching

\section{SOLAR CELL}

The generated electrical current in the semiconductor is extracted by contacts to the front and rear of the cell. The top contact structure, which allows the light to pass through it, is in the form of widely-spaced thin metal strips (usually called fingers) that supply current to a larger bus bar. The cell is covered with a thin layer of dielectric material - the antireflection coating or ARC- to minimize light reflection light reflection from the top surface. Figure 1 shows the diagram of a silicon cell, the typical solar cell in use today [4].

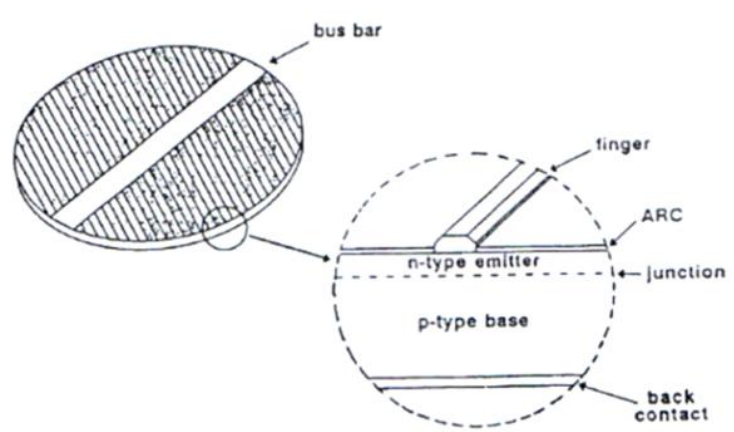

The silicon solar cell

Light generates electron-hole pairs on both sides of the junction, in the n-type emitter and in the p-type base. The generated minority carriers-electrons from the base and holes from the emitter-diffuse, within the diffusion length of the minority carriers, to the junction and are swept away by the electric field; thus the electric current across the 
device is produced. The electric currents of the electrons and holes reinforced each other since these particles carry opposite charges. Therefore the $p-n$ junction separates the carriers with opposite charge, and transforms the generation current I_ph between the bands into an electric current across the p-n junction. Three parameters are usually used to express the solar cell outputs [3]. One of these parameters is the short-circuit current I_sc which is simply the light- generated current I_ph. A second parameter is the open-circuit voltage V_oc, which is obtained by setting $\mathrm{I}=0$ in equation 1 :

$$
\mathrm{V}_{\mathrm{oc}}=\frac{K T}{q} \ln \left[\frac{I_{p h}}{I_{o}}+1\right]
$$

It is worthwhile to examine this equation in more detail. Both I_ph and I_o depend on the structure of the device. However, it is the value of I_o which can vary by many orders of magnitude, depending on the device geometry and processing that determines the open-circuit voltage in practical devices.

No power is generated under short or open circuit. The maximum power P_max produced by the device is reached at a point on the characteristic where the product I by $\mathrm{V}$ is maximum value. This is shown graphically in figure (1.4) where the position of the MPP represents the largest area of the rectangle shown. While, the third characterized parameter is the fill-factor FF which is defined as

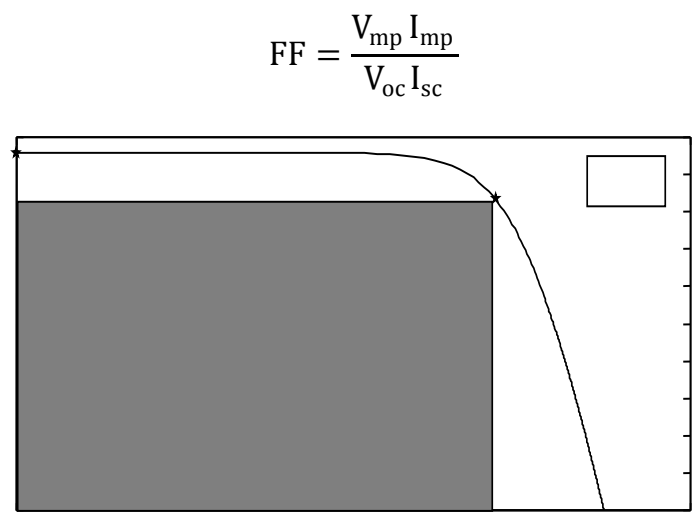

The I-V characteristic of a solar cell with the MPP

\section{FUZZY CONTROL}

Recently, fuzzy logic theory has been successfully applied to many engineering field, especially, in the field of control engineering. Practically, fuzzy logic control shows its powerful capability in dealing with those complex systems and ill-defined processes, or systems lack of knowledge of their mathematical model. This besides to it is easy to implement, as it usually needs no mathematical model of the controlled system [7].

Classical control theory, the controller is designed based on the mathematical model of the system. However, the mathematical model of the system is hard to know. So it is not easy to design a model based controller to control these complex and ill-defined system. Fortunately, human knowledge can do well in dealing with this kind of problem. It is well known that fuzzy logic provides a systematic procedure to transform a knowledge base into a practical control strategy. For a control system, the controller can be described as the relationship between the inputs and outputs. The control law of fuzzy logic controller is described by human language in the form of linguistic "IF THEN" rules. For example, "IF the speed of the car is slow, THEN accelerate it". All of these decisions are constructed according to the experience of human knowledge. The next subsections describe a general methodology for constructing an FLC. This discussion illustrates FLC components like fuzzification, defuzzification strategies, the derivation of the data base, and fuzzy control rules. Finally, the fuzzy logic is proved that human thinking does not always obey crispy logic, which is NO-YES or 0-1 logic, but is often uncertain fuzzy. This means that all things admit a certain degree of truth. The basic connective operation in conventional set theory are also applied to the fuzzy logic [5].

Considering that $\mu \mathrm{A}(\mathrm{u})$ and $\mu \mathrm{B}(\mathrm{u})$ represent the degrees of memberships of the element $u$ belongs to universe of discourse U for the fuzzy sets A and B respectively. The theoretical sets operation like union, intersection, and complement are defined for the fuzzy sets operations. These operations are considered the famous commonly used and defined via their membership functions are shown in figure (2.1) as follows.
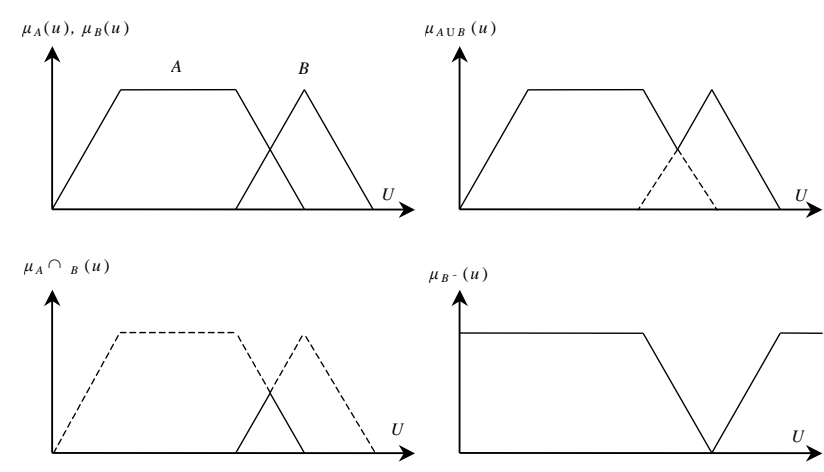

Fuzzy set operations

The mathematical model of the plant is important to design the conventional controller. The conventional controller can be designed to meet the specified performance where an accurate mathematical model is analyzed if it is possible. A reasonable good mathematical model is different to obtain, especially for complex process. If the operator of that process have good experience for controlling, it regardless of its complexity. Then, the fuzzy logic controller is very useful for complex systems which uses the experience knowledge of the operator to give good superior performance compared with conventional controller.

This means that, FLC is powerful tool especially for the processes have wide range in parameter variation [6]. The fuzzy logic controller is composed of four components are the fuzzification process, the knowledge base stage, the inference engine, and the defuzzification process. 


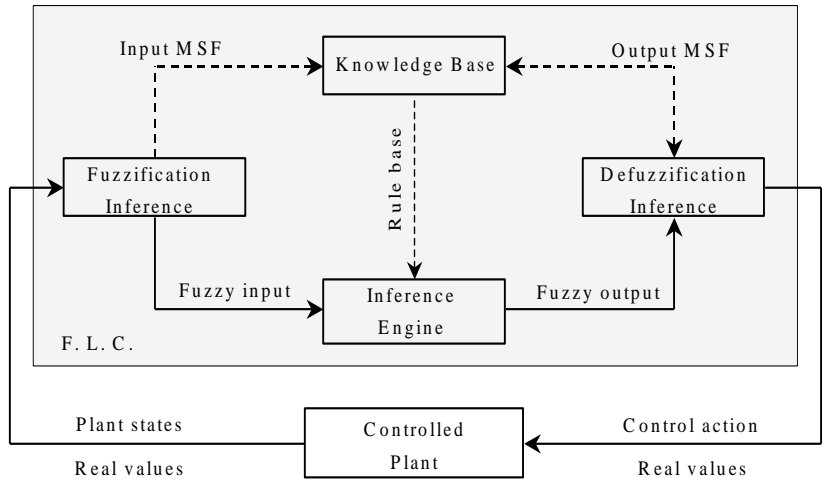

Fuzzy set operations

Since the fuzzy logic controller deals with fuzzy variables, the controlled variable of the plant must be mapped into fuzzy variable through the input membership functions. This process called fuzzification stage. That means, if the motor speed is measured and have a value of $500 \mathrm{rpm}$ as a crisp real value must be translated to the linguistic fuzzy value fast, medium, or slow [4]. Once all controlled input values are mapped into corresponding linguistic variable values, the data base and the derivation of the control rules are determined from knowledge base experience stage. The fuzzy engine inference is executed to drive a decision making from the rule base that represents the control strategy as a fuzzy output variable. The last fuzzy output variable derived from the controller also must be mapped into a corresponding real crisp value. This process is called defuzzification step which transforms the fuzzy output control action into the real crisp value of the control variable. The main four parts, which construct the components of the fuzzy logic controller will be discussed in details in the next subsections.

\section{SIMULATION RESULTS WITH SUBJECTIVE EVALUATION}

Simulation starts by considering a normal operation case. By normal operation, we mean that the system is running without exposing to any atmospheric or load variations. The atmospheric conditions are assumed to be $800 \mathrm{~W} / \mathrm{m} 2$ solar radiation and $300 \mathrm{~K}$ solar cell temperature, while the load is kept constant at. The sampling time used in simulation is $1 \mathrm{~ms}$ and the simulation run time is 0.2 second. The simulation starts from the instant at which the load is connected. Therefore, we expect that the staring simulated voltage is equal to the open circuit voltage of the PV module, while the current is, intuitively, zero, and hence the starting power is zero. Both the FLC and a PIcontroller (PIC) are applied while several gains are tried carefully for the PI-controller before obtaining its best performance where $\mathrm{KP}=0.5, \mathrm{Ti}=0.08$. Despite the short simulation period, both controllers are able to change the operating point to that at which the maximum power is delivered. Figure (4.6) shows how the maximum power condition is satisfied using both the FLC and the PIcontroller. The figure shows how the controlled variables track their optimal values accurately, and rapidly [8]. However, the PI-controller introduces some better performance indices where no overshoot is resulted and smaller integral of absolute error is obtained. It is clear that the delivered power is significantly improved comparing with the direct coupling case where an increase of $30 \mathrm{~W}$ is achieved. Thus, the delivered power obtained, using both controllers, is about $200 \%$ of its value in direct coupling case. The reason is that the DC-DC converter inserted between the PV working module and the load adjusts the resistance value seen by the PV working module when the proposed FLC or the PI-controller produces their suitable control signals and hence the appropriate duty ratio

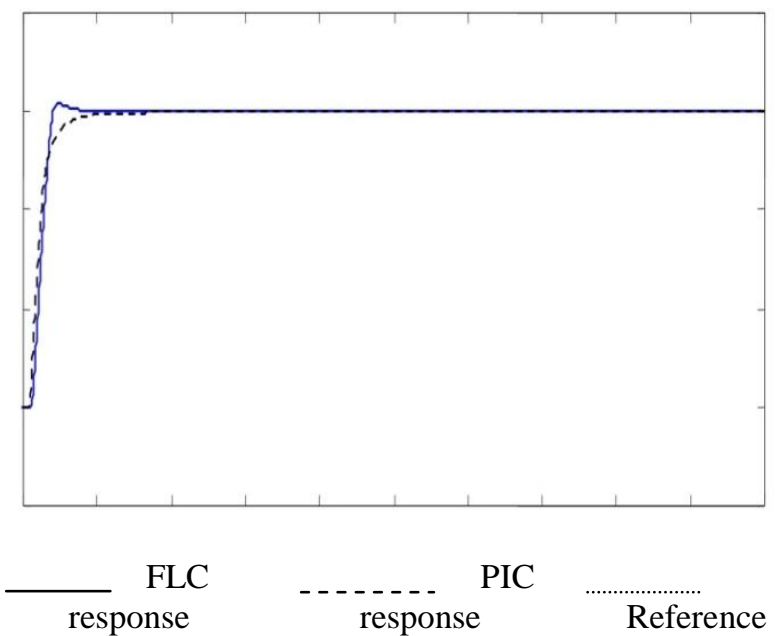

Suppose that the radiation level is changed from 700 to 900 to 700 and finally to $500 \mathrm{~W} / \mathrm{m} 2$, respectively. Assume that these variations have occurred in a short time $(0.8$ sec.), with 0.2 second separation between each variation and other [10]. These fast variations may occur in cloudy days. Although the changes in radiation levels are usually associated with relevant changes in temperature, the temperature is assumed to be constant at $300 \mathrm{~K}$, while the load has a value of. We prefer to study the effect of each variation individually and then combine all variations in one simulation case finally [9]. It is clear that both the FLC and the PI-controller introduce excellent performance where the controlled variables track their reference values exactly in a very short time. Only safe dips and jumps are recorded throughout the simulation run time. However, On the other hand, when the load is directly coupled to the working module, the voltage, the current, and hence the power, are far from their reference values since the selected load is not suitable to deliver the maximum power from the working module.

\section{CONCLUSION}

For good utilization of the PV arrays, it is necessary to operate at their maximum power points (MPP's). Therefore, many different techniques have been developed to accomplish this purpose. This project, first, introduces the basic concepts of the PV system and discusses the mismatch-problem between the PV array and the load by listing some solutions to that problem. Second, the mathematical models of the different subsystems that 
constitute the overall solar cell power supply system are derived. Then, simulation of the overall solar cell power supply system using Matlab/Simulink Software Package is presented. Finally, the simulation results are presented and conclusions of the project are extracted. To perform a subjective evaluation, the robustness of the fuzzy logic controller is tested assuming that the system is exposed to several parametric variations such as radiation level changes, solar cell temperature changes, load changes, and when subjected to all of these changes together. The fuzzy logic controller gains are accurately identified and hence it proved its ability in driving the PV module to its maximum power point in terms of majority of the performance measures. This leads to a significant increase in the delivered power, especially when the direct coupling operating point is far from the maximum power one.

\section{REFERNCES}

[1] Hohm, D. P., \& Ropp, M. E. (2003). Comparative study of maximum power point tracking algorithms. Progress in photovoltaics: Research and Applications, 11(1), 47-62.

[2] Nafeh, A. E. S. A., Fahmy, F. H., \& El-Zahab, E. M. A. (2003). Evaluation of a proper controller performance for maximum-power point tracking of a stand-alone PV system. Solar energy materials and solar cells, 75(3), 723-728.

[3] Singer, S., Rozenshtein, B., \& Surazi, S. (1984). Characterization of PV array output using a small number of measured parameters. Solar Energy, 32(5), 603-607.

[4] Krunks, M., Katerski, A., Dedova, T., Acik, I. O., \& Mere, A. (2008). Nanostructured solar cell based on spray pyrolysis deposited $\mathrm{ZnO}$ nanorod array. Solar Energy Materials and Solar Cells, 92(9), 1016-1019.

[5] Fakham, H., Lu, D., \& Francois, B. (2011). Power control design of a battery charger in a hybrid active PV generator for load-following applications. Industrial Electronics, IEEE Transactions on, 58(1), 85-94.

[6] Lee, C. C. (1990). Fuzzy logic in control systems: fuzzy logic controller. II. Systems, Man and Cybernetics, IEEE Transactions on, 20(2), 419-435.

[7] Ragot, J., \& Lamotte, M. (1993). Fuzzy logic control. International journal of systems science, 24(10), 1825-1848.

[8] Ito, S., Kitamura, T., Wada, Y., \& Yanagida, S. (2003). Facile fabrication of mesoporous $\mathrm{TiO} 2$ electrodes for dye solar cells: chemical modification and repetitive coating. Solar energy materials and solar cells, 76(1), 3-13.

[9] Farid, M. M., Khudhair, A. M., Razack, S. A. K., \& Al-Hallaj, S. (2004). A review on phase change energy storage: materials and applications. Energy conversion and management, 45(9), 15971615 .

[10] Kuznik, F., Virgone, J., \& Roux, J. J. (2008). Energetic efficiency of room wall containing PCM wallboard: A full-scale experimental investigation. Energy and buildings, 40(2), 148-156. 